\title{
PARTICLE SWARM LEARNING ALGORITHM BASED ON ADJUSTMENT OF PARAMETER AND ITS APPLICATIONS ASSESSMENT OF AGRICULTURAL PROJECTS
}

\author{
Shanlin Yang ${ }^{1}$, Weidong Zhu ${ }^{1}$, Li Chen ${ }^{1,2, *}$ \\ ${ }^{I}$ School of Management, Hefei University of Technology, Hefei ,Anhui Province, P. R. China \\ 230009 \\ 2 Department of Management Engineering, Anhui Institute of Architectural and Industry, \\ Hefei, Anhui Province, P. R. China 230022 \\ * Corresponding author, Address: Department of The Management Engineering, Anhui \\ Institute of Architectural and Industry, Hefei 230022, Anhui Province, P. R. China, Tel: \\ 13339296584, Fax: 0551-3828127,Email: chinalichina@163.com
}

Abstract: The particle swarm, which optimizes neural networks, has overcome its disadvantage of slow convergent speed and shortcoming of local optimum. The parameter that the particle swarm optimization relates to is not much. But it has strongly sensitivity to the parameter. In this paper, we applied PSO-BP to evaluate the environmental effect of an agricultural project, and researched application and Particle Swarm learning algorithm based on adjustment of parameter. This paper, we use MATLAB language .The particle number is 5, $30,50,90$, and the inertia weight is $0.4,0.6$, and 0.8 separately. Calculate 10 times under each same parameter, and analyze the influence under the same parameter. Result is indicated that the number of particles is in $25 \sim 30$ and the inertia weight is in $0.6 \sim 0.7$, and the result of optimization is satisfied.

Keywords: parameter, the particle swarm optimization, agricultural projects measurement

\section{INTRODUCTION}

Artificial neural network (ANN) is a rising borderline science. Compared to the mathematical statistics, Artificial neural network doesn't need exact

\footnotetext{
Please use the following format when citing this chapter:

Yang, S., Zhu, W. and Chen, L., 2009, in IFIP International Federation for Information Processing, Volume 294, Computer and Computing Technologies in Agriculture II, Volume 2, eds. D. Li, Z. Chunjiang, (Boston: Springer), pp. 1379-1388.
} 
mathematical model and it can solve some problems that traditional statistical methods failed to resolve. Up to now, various types of ANN have been developed in order to address different problems, such as classification, optimization, pattern recognition, data reduction, control and prediction. About $80 \%-90 \%$ percent of ANN is BP network or its change form. BP neural network is one of the most widely used neural networks, and it is a kind of multi-layer back propagation neuron network. It contains three layers: the input layer, the output layer, and the hidden layers. Its output is continuous variable from 0 to 1 and it can realize any nonlinear mapping. The BP network application in agriculture project includes: (1) Modeling and simulation of post-evaluation based on rough set-neural network. (2) Application of rough neural network in agricultural engineering project evaluation. (3) Appraise a model of agricultural high sci-tech agriculture projects base on BP (Chen Li et al.,2006; Chen Li, ZHU Wei-dong,2006; Chui W F et al.,2006).

There are a lot of neural network train methods, but there are few defects, such as it is slow to disappear, easy to converge to the local extreme point. BP is difficult for the function to get out when it gets into a local extreme point. Too many nodes in the hidden layer will lead to the long time of network learning, even the failure of convergence. An overfit phenomenon exists in the BP network (Li Xiaoqing,2006).

In order to overcome this shortcoming of BP, in this paper, we improve BP and adopt the method of Particle Swarm learning algorithm based on adjustment of parameter and Its Applications assessment of agricultural projects.

The PSO, one kind of swarm intelligence, is proposed by Kennedy and Eberhart in 1995. The underlying motivation for the development of PSO algorithm was animal social behavior such as fish schooling, birds flocking, schooling, and swarming. Some of the attractive features of the PSO include the ease of implementation and the fact that no gradient information is required (LIU Yi-jian, et al.,2005).

PSO is the simulation process of birds looking for food. During the birds' looking for food, they adjust the flight direction and speed based on external information at any time. Each bird will be regarded as a non-cubage particle (Cui Guang-zhao, et al.,2007).

Initially, a population of $n$ particles is randomly generated in the particle swarm optimization algorithm and searches for optima by updating generations. Particle swarms have two primary operators: velocity update and position update. The swarm direction of a particle is defined by the set of particles neighboring the particle and its history experience. During flight, each particle adjusts its position according to its own experience, and the experience of neighboring particles, making use of the best position encountered by itself and its neighbors (PAN Hong-xia, et al.,2006). 
PSO is a generic heuristic optimization algorithm based on the concept of swarm intelligence. It requires less computation times and less memory. Till now PSO has fewer parameters to adjust, and it has been used to solve many engineering and economic problems. PSO as the data analysis tool of a kind of complicated non-linear course, has already extensively applied in patternrecognition, knowledge engineering, trend analysis, ect. However, it has not been employed so far in an environmental assessment of agricultural project. Here, we apply the adapted PSO approach to search for good environmental assessment of agricultural project sequences with fitness function (Zhao Bo, et al.,2004).

\section{PARTICLE SWARM OPTIMIZATION ALGORITHM}

\subsection{Mathematical models of PSO algorithm}

Particle swarm optimization is a member of the wide category of swarm intelligence methods that traces its evolution to the emergent motion of a flock of birds searching for food. It uses a number of particles that constitute a swarm. Each particle traverses the search space, looking for the global minimum (or maximum). During each generation, each particle moves in the search space with a velocity according to its own previous best solution and its group's previous best solution.

The basic particle swarm model consists of an initial swarm of random particles. At the beginning, the PSO algorithm randomly initializes the population (called swarm) of individuals. If there are $m$ particles which conclude a group in $D$-dimensional space, the $i$ th particle is $X_{i}=\left(x_{i 1}, x_{i 2}, \ldots\right.$, $\left.x_{i D}\right)$, where $(i=1,2, \ldots, m)$, that is, the position of the $i$ th particle in dimensional space is $X_{i}$. While searching optimal solution in search space, each particle remembers two variables : one is the best position found by its own so far, denoted by pbest and another is the best position among all particles in the swarm, denoted by gbest. The $i$ th particle's flying speed can be noted as $V_{i}=\left(v_{i 1}, v_{i 2}, \ldots, v_{i D}\right)$. (LI Qiang et al.,2007).

Each particle updates its own velocity and position according to formula Equ.( 1 ) and Equ.( 2 )

$$
\begin{aligned}
& v_{i d}^{n+1}=w v_{i d}^{n}+c_{1} r_{1}^{n}\left(p_{i d}^{n}-x_{i d}^{n}\right)+c_{2} r_{2}^{n}\left(p_{g d}^{n}-x_{i d}^{n}\right) \\
& x_{i d}^{n+1}=x_{i d}^{n}+v_{i d}^{n+1}
\end{aligned}
$$


where $x_{i d}^{n+1}$ is the current position of Particle $\mathrm{i} ; p_{i d}$ is the searched optimal position of Particle i ; $p_{g d}$ is the searched optimal position of the whole particle swarm . In the above equations, $c_{1}$ is called self-confidence range; $c_{2}$ is called swarm range, and they pull each particle towards pbest and gbest positions; $r_{1}$ and $r_{2}$ are randomly generated value between 0 and 1 . They determine the affection of $p_{i d}$ and $p_{g d}$, the relative influences of the social and the cognition components; $w$ is the inertial weight factor, which is able to adjust the abilities of overall and local search; $v_{i d}^{n+1}$ the velocity of the $i$ th particle, must lie in the range $\left[v_{\mathrm{d} \min }, v_{\mathrm{d} \max }\right] ; v_{\mathrm{d} \max }$ represents for the fast iterative speed substitution calculation; it is a constant set by the user. Large values of $v_{i d}^{n+1}$ can result in particles moving past good solutions, while small values can result in insufficient exploration of the search space (YANG Huachao et al.,2007) .

\subsection{PSO algorithm procedures}

The whole process of the proposed algorithm can be described as follows:

(1) Randomly generate particles and the particle velocities; Initialize particle positions and velocities.

(2) Evaluate its degree of adaptability to the particle swarm. Calculate all the fitness values of each particle.

(3) Compare each individual's evaluation value with its pbest. The best evaluation value among the pbest's is denoted as gbest. Update pbest and then gbest based on the values. If the new value is better than the previous pbest, the new value is set to be pbest.

(4) Modify the member velocity of each individual $P_{\mathrm{g}}$ according to Eqs.(1) and Eqs. (2).

(5) If the stopping criteria are met, the positions of particles represented by gbest are the optimal solution. If the result is satisfied with the number of iteration or precision, then terminate the calculation, or else return to the step (2).

\subsection{Analysis on inertia weight in particle swarm optimization}

Particle Swarm Optimization (PSO) is simple and easy to implement, and it can generate high-quality solutions within shorter calculation time and more stable convergence characteristic than other stochastic methods. There are not many parameters which need to be tuned in PSO. Although the dimension of particles and the range of particles is important factors in 
particle swarm optimization. Here, we focus on the influence of the number of particles and the inertia weight.

The inertia weight is the crucial parameter of the particle swarm optimization. It controls the impact of the previous history of velocities on the current velocity. Suitable selection of inertia weight $w$ provides a balance between global and local explorations, thus requiring less iteration on average to find a sufficiently optimal solution. The inertia weight is an important parameter of the PSO choice which can be divided into two categories: fixed inertia weight and time variant inertia weight. The choice of a fixed inertia weight is to choose a constant value as a weight in the process, and it is unchanged in the optimization.

The time variant inertia weight is selected a certain range. For example, Here, a linearly iteration decreasing $w$ is used, which is usually employed as a trade off between the global and local exploration abilities of the swarm. The general inertia weight linearly descend with the number of iterations in common. It is better to initially set the inertia weight to a large value, in order to promote global exploration of the search space, and gradually decreases it to get more refined solutions. Thus the inertia weight can be described as

$$
\mathrm{w}=\mathrm{wmax}-[(\mathrm{wmax}-\mathrm{wmin}) / \text { itermax }] \cdot \text { iter }
$$

In the equation, where $w_{\max }$ and $w_{\min }$ denote the maximum and minimum value of the inertia weight; iter $_{\max }$ denote the maximum number of iterations, and iter is the current number of iterations. For example, the parameters of PSO algorithm are selected as $w=1.5 \sim 0.2$, which means that $w$ starts from 1.5 and gradually decreases to 0.2 .

In this paper, we supposed the weights are fixed inertia weight. Usually, larger $w$ is expected to facilitate the global exploration at the beginning of the run, while smaller $w$ is expected to facilitate the local exploration near the end of the run. The initial positions and velocities of all particles are generated randomly in the search space. Then, the processes from Eqs. (1) to Eqs. (3) are repeated until a user defined stopping criterion is reached.

\subsection{Artifical experiments}

\subsubsection{Establish index system of an environmental assessment of agricultural project}

In this paper, we use an agricultural project as an example and utilize PSO$\mathrm{BP}$ to evaluate environmental-quality assessment. We establish the index system of an environmental assessment of agricultural project. The index we 
select here is mainly reflect environmental benefits, environmental pollution and ecological degradation ( Chen li ,Zhu Weidong, 2008) (Table 1.).

Table 1. Index system of an environmental assessment of agricultural project

\begin{tabular}{cl}
\hline \multicolumn{1}{c}{ Index } & \multicolumn{1}{c}{ Contents } \\
\hline \multirow{2}{*}{ Environmental benefits } & Grassplot area rate \\
\cline { 2 - 2 } Environmental pollution & Nursery stock area \\
\cline { 2 - 2 } & The agricultural product contaminates \\
& Soil contaminates \\
\cline { 2 - 2 } Ecocide & Water body pollution \\
\hline & The vegetation covers lessening rate \\
& Apply chemical \\
& Loss of soil and water area \\
\hline
\end{tabular}

\subsubsection{Application and Particle Swarm learning algorithm based on adjustment of parameter}

To demonstrate the good performance of PSO, PSO-BP is also used to find the optimal parameter combination. However, the computation time using PSO-BP is much less than using BP. So, it is much faster using PSO$\mathrm{BP}$ than BP. In this paper, we do not compare the computation time using PSO-BP and BP.

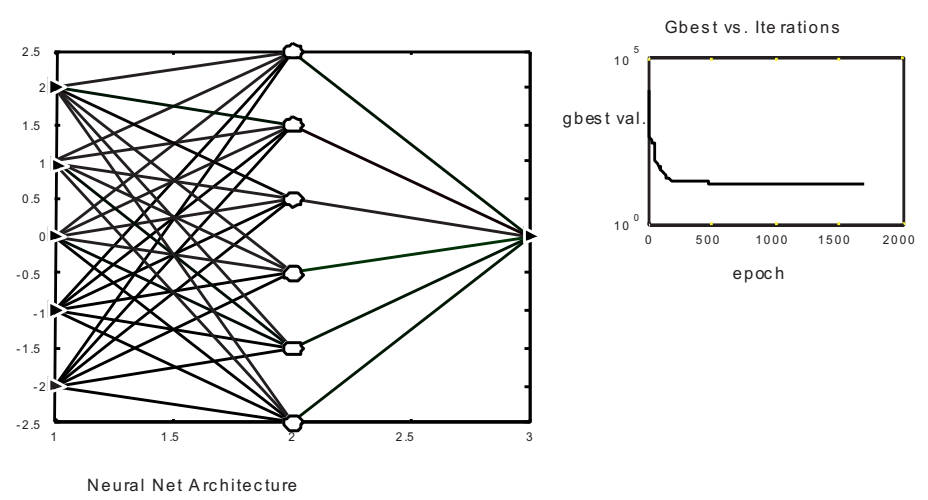

PSO: $1 / 2000$ iterations, GBest $=10091.998215630534$.
PSO: $500 / 2000$ iterations, GBest $=17.466520066108341$.
PSO: $1000 / 2000$ iterations, GBest $=17.433778425823558$.
PSO: $1500 / 2000$ iterations, GBest $=17.433623293802363$.
PSO: $1600 / 2000$ iterations, GBest $=17.433623293802359$.
PSO: $1691 / 2000$ iterations, GBest $=17.433623293802359$.
SSE $=2.3187 \mathrm{e}+003$
$-->$ Solution likely, GBest hasn't changed by at least $1 \mathrm{e}-009$ for 400 epochs.

Fig. 1. Neural Net Architecture and Optimize process

The proposed PSO based approach was implemented by using the MATLAB language and the experiments have been carried on two infrared 
images using MATLAB7.0 on $2.0 \mathrm{GHz}$ Pentium 4 PC. Initially, several runs have been done with different values of the PSO. Meanwhile, several experiments have been done in order to obtain the penalty parameters.

In the neural network algorithm trained by particle swarm optimization: Parameters in PSO algorithm are described as follows: the maximum allowed iteration number is 2000 ; the acceleration constants are selected as the default values $c_{1}=c_{2}=2$.SSE is equivalent to $2.3187 \mathrm{e}+003$; The segmentation results are shown in Fig1.

Tables $2 \sim 4$ show the preliminary results of the comparisons. Each test is run 10 times and each run Loops 2000 generations.

Table 2. Comparison of experimental result

\begin{tabular}{|c|c|c|c|c|c|c|c|}
\hline \multirow{2}{*}{ No. } & \multirow{2}{*}{$\begin{array}{c}\text { Particle number } \\
\text { Weight }\end{array}$} & \multicolumn{3}{|c|}{5} & \multicolumn{3}{|c|}{30} \\
\hline & & 0.4 & 0.6 & 0.8 & 0.4 & 0.6 & 0.8 \\
\hline 1 & \multirow{10}{*}{ ESS } & 662.613 & 1860.8 & 201.626 & 0.235 & 0.423 & 0.340 \\
\hline 2 & & 1594.7 & 1.701 & 1242.0 & 0.332 & 0.248 & 0.507 \\
\hline 3 & & 129.401 & 1.826 & 2212.8 & 0.234 & 0.273 & 0.206 \\
\hline 4 & & 824.866 & 3819.1 & 748.385 & 0.186 & 0.256 & 0.210 \\
\hline 5 & & 654.370 & 1072.4 & 590.781 & 0.240 & 0.408 & 0.288 \\
\hline 6 & & 83.344 & 160.128 & 15.950 & 0.464 & 0.276 & 0.800 \\
\hline 7 & & 2231.7 & 1.423 & 19597 & 0.340 & 0.533 & 0.669 \\
\hline 8 & & 81.685 & 16.514 & 4.512 & 0.426 & 0.241 & 0.629 \\
\hline 9 & & 2318.7 & 41.596 & 267.348 & 0.353 & 0.246 & 0.508 \\
\hline 10 & & 11.648 & 5.275 & 0.777 & 0.467 & 0.496 & 0.506 \\
\hline \multirow{2}{*}{ No. } & Particle number & & 50 & & & 90 & \\
\hline & Weight & 0.4 & 0.6 & 0.8 & 0.4 & 0.6 & 0.8 \\
\hline 1 & \multirow{10}{*}{ ESS } & 0.233 & 0.644 & 0.205 & 0.224 & 0.215 & 0.373 \\
\hline 2 & & 0.286 & 0.303 & 0.283 & 0.265 & 0.242 & 0.399 \\
\hline 3 & & 0.458 & 0.398 & 0.255 & 0.289 & 0.285 & 0.238 \\
\hline 4 & & 0.130 & 0.523 & 0.270 & 0.150 & 0.174 & 0.340 \\
\hline 5 & & 0.279 & 0.250 & 0.344 & 0.236 & 0.159 & 0.219 \\
\hline 6 & & 0.307 & 0.202 & 0.349 & 0.122 & 0.436 & 0.256 \\
\hline 7 & & 0.154 & 0.139 & 0.302 & 0.495 & 0.323 & 0.218 \\
\hline 8 & & 0.347 & 0.504 & 0.481 & 0.420 & 0.358 & 0.388 \\
\hline 9 & & 0.346 & 0.266 & 0.308 & 0.288 & 0.378 & 0.375 \\
\hline 10 & & 0.390 & 0.358 & 0.226 & 0.258 & 0.413 & 0.220 \\
\hline
\end{tabular}

Sufficient experiments have been done on inertia weight that it is an important parameter in the algorithm. The particle swarm, which optimizes neural networks, has overcome its disadvantage of slow convergent speed and shortcoming of local optimum. The parameter that the particle swarm optimization relates to is not much. But the particle swarm optimization is strongly sensitive to the parameter.

When the particle number is 5, 30, 50, 90 separately, and the inertia weight is $0.4,0.6,0.8$ separately, calculate 10 times under the condition of 
each same parameter. Analyze the influence of the parameter. The acceleration constants are selected as the default values $c_{1}=c_{2}=2$.The inertia weight $w$ is $0.4,0.6$, and 0.8 , respectively. The SSE of optimization with PSO by changing inertia weight is listed in Table 2.

The Optimum value and the poorest value of the target satellite are listed in Table 3. Results of optimization with PSO by changing inertia weight are listed in Table 4.

Table 3. Results of optimization with PSO

\begin{tabular}{ccccccc}
\hline \multirow{2}{*}{$\begin{array}{c}\text { Particle } \\
\text { Number }\end{array}$} & \multicolumn{2}{c}{ Inertia Weight 0.4} & \multicolumn{2}{c}{ Inertia Weight 0.6} & \multicolumn{2}{c}{ Inertia Weight 0.8} \\
\cline { 2 - 6 } & Optimum alue & Poorest value & Optimum alue & Poorest value & Optimum value & Poorest value \\
\hline 5 & 11.648 & 2231.7 & 1.423 & 3819.1 & 0.7778 & 19597 \\
30 & 0.1865 & 0.4674 & 0.2412 & 0.5337 & 0.2062 & 0.8000 \\
50 & 0.1308 & 0.4582 & 0.1390 & 0.6446 & 0.2052 & 0.4817 \\
90 & 0.1223 & 0.4957 & 0.1599 & 0.4367 & 0.2182 & 0.3979 \\
\hline
\end{tabular}

Table 4. Results of optimization with PSO by changing weight

\begin{tabular}{cccccccccc}
\hline \multirow{2}{*}{ Weight } & \multicolumn{3}{c}{ Particle number 20 } & \multicolumn{3}{c}{ Particle number 25 } & \multicolumn{3}{c}{ Particle number 28 } \\
\cline { 2 - 10 } & \multicolumn{3}{c}{ ESS } \\
\hline 0.4 & 0.630 & 0.6364 & 0.4818 & 0.2291 & 0.5244 & 0.6727 & 0.1326 & 0.7571 & 0.450 \\
0.5 & 1.355 & 0.4219 & 0.3700 & 0.4812 & 0.429 & 0.3823 & 0.1926 & 0.5680 & 0.130 \\
0.6 & 0.498 & 0.4624 & 0.3505 & 0.3489 & 0.3864 & 0.5889 & 0.3782 & 0.1982 & 0.347 \\
0.7 & 0.595 & 0.3869 & 0.2984 & 0.6638 & 0.4863 & 0.4161 & 0.5681 & 0.1187 & 0.314 \\
0.8 & 0.321 & 0.5116 & 0.3994 & 0.4028 & 0.2502 & 0.4951 & 0.4101 & 0.7096 & 0.156 \\
0.9 & 0.905 & 0.6007 & 0.5599 & 0.8496 & 0.8480 & 1.2726 & 0.4405 & 0.6945 & 0.282 \\
\hline
\end{tabular}

\subsubsection{Experiments analysis}

Analysis from Table 2, Table 3, and Table 4, we can conclude:

(1) We test each sample with 10 times for impartial results and treat the average of each sample as the final result. In the test, all the algorithms run 10 times with 2000 iterations. When the swarm size particle number is fewer, calculating under the same condition ten times, the SSE of optimization with PSO fluctuates greatly. When the number of the particle is 5 , and the inertia weight is 0.8 , and optimum value of the ten times is 0.7778 . The worst value is 19597 . When the number of the particle is greater than 20, calculating under the same condition, the SSE of optimization with PSO fluctuates not greatly (Table 2 .).

(2) In Fig.1, the PSO algorithm converges fast at the beginning of the run but slows down when it gets close to the global optimum. It has overcome its disadvantage of local optimum when the inertia weight is greater, but it has the relatively slow convergent speed.

(3) Final optimization results of the algorithm are shown in Table 2. In the test, all the algorithms run 10 times with 2000 iterations.

From the result of PSO operation of a agriculture project, we find that 590 particles are used in PSO, which is a balance between the accuracy 
From the result of the applications assessment of agricultural projects

based on the PSO, we find that although only few parameters are required, they are important for the optimization efficiency of particle swarm optimization. The inertia weight and the

required in search of the global optimum and time consumed (Table 3.). Through being ten experiments, result is indicated that the number of particles is in $25 \sim 30$ and the inertia weight is in $0.6 \sim 0.7$, and the result of optimization is satisfied. When the inertia weight is too great, such as 0.9 , the result is not pretty good. (Table 4.).

The result of this paper has confirmed that the reliability of the PSO algorithm is more powerful in the aspects of an environmental assessment of agricultural project. PSO algorithm has been successfully applied to solve the environmental assessment of agricultural project.

\section{CONCLUSION}

From the result of the applications assessment of agricultural projects based on the PSO, we find that although only few parameters are required, they are important for the optimization efficiency of particle swarm optimization. The inertia weight and the number of PSO are the crucial parameters of the particle swarm optimization.

Results indicated that when the inertia weight is smaller, especially when the particle is fewer, it is fast convergent speed at this moment, but apt to fall into local optimum. The larger the number of particles adopted in PSO, the fewer the opportunities to be trapped in suboptima. It finds particle counts between 25-30, while weight in 0.6-0.7, and it optimizes that result is getting more ideal.

The results of this paper confirmed that the reliability of the PSO algorithm is more powerful in the aspects of an environmental assessment of agricultural project.

PSO-BP neural network can be used to predict PSO Applications assessment of agricultural projects based on adjustment of parameters, which not only overcomes the long time of network learning, but also avoids the failure of convergence. No more than $2.3187 \mathrm{e}+003$ maximum errors showed that the model predicted the PSO Applications assessment of agricultural projects with highly accuracy.

The application field and the theory about PSO still need exploring. The results of this paper confirmed that the reliability of the PSO algorithm is more powerful in the aspects of an environmental assessment of agricultural project. Comparison functions adopted here are four benchmark functions used by many researchers. They are the Sphere, Griewank, Rastrigrin and Rosebrock. How to advance the accuracy of the results by inducing other optimization methods and how to make our method be applied in practice is 
future research for us. It should be noted that the validity of the proposed method in this study is limited within the cases where inertia weight is fixed. The time variant inertia weight and the four benchmark functions mentioned above should be addressed in the future.

\section{REFERENCES}

Chen li, Zhu Weidong,The enviromental quality assessment of neural network algorithm trained by particle swarm optimization, Acta Ecologica Sinica,Vol.28,No.3, 2008(3):10721079

Chen Li, Zhu Weidong. Application of rough neural network in agricultural engineering project evaluation . Transactions of the CSAE, 2006, 22 (7): 230- 232

Chen Li, Zhu Weidong. Modeling and Simulation of Post-evaluation Based on Rough SetNeural Network. Journal of System Simulation, 2006,18 (8):2158-2161

Chui W F, Huo X X,Zhuang S H,etc. Appraise a model of agricultural high sci-tech agriculture projects base on BP. Jour. of Northwest Sci-Tech Univ. of Agri and For. (N at. Sci.Ed. ), 2006,34(7) :160-164

Cui Guangzhou, Niu Yunyun, Wang Yanfeng, Zhang Xuncai and Pan Linqiang. A new approach based on PSO algorithm to find good computational encoding sequences, Progress in Natural Science, Vol. 17, No. 6, 2007(6):712-716

Li Qiang, Guo Fucheng, Zhou Yiyu. A New Satellite Passive Locating Algorithm Using Frequency-only Measurements Based on PSO, Journal of Astronautics, Vol. 28, No. 6,2007(6):1575-1582

Li Xiaoqing. Design optimization based on neural network and genetic algorithm, Computer Automated Measurement \& Control,2006, 14 (2):253-255 (in Chinese)

Liu Yijian, Zhang Jianming, Wang Shuqing. Parameter estimation of cutting tool temperature nonlinear model using PSO algorithm, Journal of Zhejiang University Science, 2005,6(10):1026-1029

Pan Hongxia, Ma Qingfeng. Research on Gear-box Fault Diagnosis Method Based on Adjusting-learning-rate PSO Neural Network, Journal of Donghua University (Eng. Ed. ),Vol . 23, No. 6, 2006(10):29-32

Yang Huachao, Zhang Shubi, Deng Kazhong, Du Peijun, Research into a Feature Selection Method for Hyperspectral Imagery Using PSO and SVM, Journal of China University of Mining \& Technology, Vol.17, No.4, 2007(12):473-478

Zhao Bo, Guo Chuangxin, Cao Yijia. Optimal Power Flow Using Particle Swarm Optimization and Non-Stationary Multi-Stage Assignment Penalty Function, Transactions of China Electro Technical Society, Vol.19, No.5, 2004 (5):47-53 